\title{
SISTEMA JURÍDICO, PRINCIPIOS JURÍDICOS Y RAZÓN PRÁCTICA *
}

\section{Introducción}

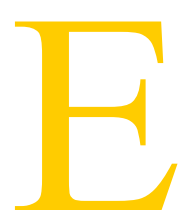

xiste para todo caso jurídico una única respuesta correcta? Esta pregunta formula uno de los problemas más discutidos de la actual filosofía del Derecho. Quien desencadenó la discusión fue el filósofo del Derecho de Oxford Ronald Dworkin. La tesis de Dworkin de que existe una única respuesta correcta para cada caso se incluye en una teoría de los sistemas jurídicos que se distingue fundamentalmente de teorías positivistas como las de Hart y Kelsen. Según la perspectiva positivista, el sistema jurídico es, al menos en lo esencial, un sistema de reglas que se pueden identificar como reglas jurídicas sobre la base de su validez y/o eficacia. Un tal sistema jurídico es siempre, por diversos motivos, un sistema abierto; sobre todo, por causa de la vaguedad del lenguaje del Derecho, la posibilidad de conflictos entre normas y la existencia de casos no regulados. Si un caso cae en un espacio vacío del sistema jurídico que no puede tampoco ser llenado en forma intersubjetivamente obligatoria con ayuda de la metodología jurídica, entonces por definición el juez no está vinculado por el sistema jurídico. Debe decidir por medio de fundamentos extrajurídicos. Su situación se asemeja en todo a la del legislador. En este caso no se puede hablar de una única respuesta correcta ya dada por el sistema jurídico, que sólo cabe reconocer.

Dworkin contrapone a este modelo de reglas del sistema jurídico un modelo de principios. Según el modelo de principios, el sistema jurídico está compuesto, además de por reglas, de un modo esencial, por principios jurídicos. Los principios jurídicos deben permitir que también exista una única respuesta correcta en los casos en que las reglas no determinan una única respuesta

${ }^{*}$ Este texto integra la ponencia presentada por el autor en las IV Jornadas Internacionales de Lógica e Informática Jurídicas, celebradas en San Sebastián en septiembre de 1988. 
correcta. La única respuesta correcta o verdadera sería así la que mejor pueda justificarse a través de una teoría substantiva que contenga aquellos principios y ponderaciones de principios que mejor se correspondan con la constitución, las reglas de Derecho y los precedentes. Dworkin reconoce que con esto no se ha encontrado ningún procedimiento que muestre necesariamente la única respuesta correcta. Sin embargo, ello no constituye una objeción en contra de su existencia. Un juez ideal, al que Dworkin llama «Hércules», equipado con «superman skill, learning, patience and acumen», es decir, con habilidad, sabiduría, paciencia y agudeza sobrehumanas, estaría en situación de encontrar la única respuesta correcta. Al juez real le corresponde la tarea de aproximarse a este ideal lo más posible.

La teoría de Dworkin de una única respuesta correcta plantea una gran cantidad de cuestiones. Aquí sólo cabe considerar una parte de ellas. Mi intervención se dividirá en dos partes. El objeto de la primera parte es la teoría de los principios. Se tratará de mostrar que los criterios de Dworkin para la distinción entre reglas y principios afectan ciertamente a puntos importantes, pero no al núcleo. Si se los analiza a fondo, queda claro que una teoría de los principios por sí sola no está en condiciones de sostener la tesis de la única respuesta correcta, lo que, sin embargo, no hace que disminuya su importancia para la filosofía jurídica, la metodología jurídica y la dogmática jurídica. En la segunda parte pretendo captar los déficits de la teoría de los principios a través de una teoría de la argumentación jurídica orientada de acuerdo con el concepto de razón práctica. La unión de ambas teorías constituye la base de una teoría del Derecho en la que rige una versión débil de la tesis de la única respuesta correcta.

\section{Teoría de los principios}

\section{1. Reglas y principios}

Tanto las reglas como los principios pueden concebirse como normas. En tal caso, de lo que se trata es de una distinción dentro de la clase de las normas. Los criterios de distinción que se ofrecen son numerosos y de diverso tipo.

\section{1. 1. La generalidad como criterio de distinción}

El criterio de distinción más frecuentemente citado es el de la generalidad. Según ello, los principios son normas de un grado 
de generalidad relativamente alto, y las reglas, normas de un grado relativamente bajo. Un ejemplo de norma de un grado relativamente alto de generalidad es la norma de que todos gozan de libertad de creencias. En cambio, tiene un grado relativamente bajo de generalidad una norma que dice que todo recluso tiene el derecho de apartar a otros reclusos de sus creencias. Quien considera la generalidad como decisiva llega a la conclusión de que entre las reglas y los principios existe sólo una distinción de grado. Esto puede designarse como la «tesis débil de la separación». La tesis fuerte de la separación la representa, en cambio, quien afirma que la diferencia entre reglas y principios no es simplemente de grado, sino de tipo cualitativo. Esta tesis es correcta. Queda por saber cómo hay que fundamentarla.

\section{1. 2. Los criterios de distinción de Dworkin}

Dworkin emplea dos argumentos. El primero dice que las reglas son aplicables en la forma todo-o-nada (all-or-nothing-fashion), pero en cambio los principios no. Si se da el supuesto de hecho de una regla, existen sólo dos posibilidades. O la regla es válida, y entonces deben aceptarse las consecuencias jurídicas, o no es válida, y entonces no cuenta para nada en la decisión. En cambio los principios, aun cuando según su formulación sean aplicables al caso, no determinan necesariamente la decisión, sino que solamente proporcionan razones que hablan en favor de una u otra decisión. El segundo argumento, vinculado con el anterior, hace valer que los principios tienen una dimensión que las reglas no exhiben, es decir, una dimensión de peso (dimension of weight) que se muestra en las colisiones entre principios. Si colisionan dos principios, se da un valor decisorio al principio que en el caso de colisión tenga un peso relativamente mayor, sin que por ello quede invalidado el principio con el peso relativamente menor. En otros contextos, el peso podría estar repartido de manera opuesta. En cambio, en un conflicto entre reglas que sucede, por ejemplo, cuando una regla manda algo y otra prohíbe lo mismo, sin que una regla establezca una excepción para la otra, al menos una debe siempre ser inválida.

Puede dudarse de la solidez del argumento todo-o-nada si se tiene en cuenta la posibilidad de que con ocasión de la decisión de un caso se introduzca en una regla una nueva excepción hasta entonces desconocida. Pero en tal caso, el segundo argumento de Dworkin, la tesis de que los conflictos de reglas ofrecen una 
estructura enteramente distinta a la de las colisiones entre principios, ofrece un criterio de distinción adecuado. Esta tesis lleva además directamente a un punto decisivo para la teoría de los principios. Por ello se analiza más detenidamente en relación con dos decisiones del Tribunal Constitucional alemán.

2. 1. 3. Conflictos de reglas y colisiones de principios como puntos de apoyo para la obtención de criterios de distinción adecuados

En la primera decisión se trata de un conflicto entre una norma jurídica del Estado federal (Bund) y una norma de un land ${ }^{1}$. La norma jurídica del land prohíbe la apertura de puestos de venta los miércoles desde las 13 horas, mientras que la norma federal lo permite hasta las 19 horas. El tribunal resuelve este caso según la norma de conflicto «El Derecho federal prevalece sobre el Derecho del land» (Art. 31 de la Ley Fundamental), mientras que declara nula la norma jurídica del land. Este es un caso clásico de un conflicto de reglas. Las dos normas son contradictorias. Una permite lo que la otra prohíbe. Si ambas fueran válidas, la apertura los miércoles por la tarde estaría tanto permitida como prohibida. La contradicción se elimina declarando a una norma nula, por tanto, no válida, y expulsándola así del orden jurídico.

De manera enteramente distinta procede el tribunal en una decisión sobre la celebración de un juicio oral contra un inculpado al que amenaza el peligro de un ataque de apoplejía y un infarto ${ }^{2}$. Las normas en colisión son, por un lado, la norma del Art. 2, ap. 2, párr. 1 de la Ley Fundamental, que reconoce al individuo un derecho fundamental a la vida y a la integridad física, y, por otro lado, el principio del Estado de Derecho, en cuanto que impone al Estado el deber de garantizar un eficiente funcionamiento de las instituciones del Derecho penal. Si existiera sólo el derecho fundamental, entonces la celebración de un juicio oral que pusiera en peligro la vida y la salud de un inculpado habría que calificarla sencillamente de prohibida. Si existiera sólo el deber del Estado de cuidar de un eficiente funcionamiento de las instituciones jurídicas, entonces habría que considerar el juicio oral simplemente como obligatorio o, cuando menos, permitido. El tribunal habría, por tanto, podido resolver

\footnotetext{
${ }^{1}$ BverfGE, 1, 283 (292).

${ }^{2}$ BverfGE, 51, 324.
} 
el caso eliminando del ordenamiento jurídico bien el derecho fundamental, o bien el deber. Si hubiera hecho esto habría tratado la colisión de las correspondientes normas como una contradicción y, por ello, como un conflicto de reglas. El proceder del tribunal sin embargo es de un tipo enteramente distinto. No habla de una contradicción entre ambas normas, sino de una tensión, y subraya que ninguna de ellas goza «simplemente de primacía frente a la otra», lo que sería el caso si una de las normas, como en el conflicto de reglas, hubiese sido declarada inválida. El caso ha de ser resuelto más bien a través de una ponderación, con lo cual todo depende de si «el interés del inculpado contrario a la celebración, en el caso concreto pesa de un modo claro esencialmente más que los intereses a cuya protección debe servir la medida estatal». Este es un caso prototípico de colisión de principios. El factor decisivo lo constituye el principio al que le corresponde un peso relativamente mayor en el caso concreto. Al principio que juega en sentido contrario se le hace retroceder, pero no se le declara inválido,

\section{1. 4. El núcleo de la diferencia entre reglas y principios}

Podría pensarse que con ello se ha dicho todo lo esencial sobre la estructura lógica de los principios, y que ahora, sobre esta base, podría comprobarse la corrección de la tesis de Dworkin de la única respuesta correcta. Sin embargo, esto sería precipitado. Se podría preguntar por qué colisionan los principios de la manera descrita. Sólo esta cuestión, no planteada por Dworkin, lleva al núcleo de la diferencia entre reglas y principios. Si no se capta esto, difícilmente puede esperarse extraer las conclusiones correctas de la presencia en el orden jurídico tanto de reglas como de principios.

El punto decisivo para la distinción entre reglas y principios es que los principios son normas que ordenan que se realice algo en la mayor medida posible, en relación con las posibilidades jurídicas y fácticas. Los principios son, por consiguiente, mandatos de optimización que se caracterizan porque pueden ser cumplidos en diversos grados y porque la medida ordenada de su cumplimiento no sólo depende de las posibilidades fácticas, sino también de las posibilidades jurídicas. El campo de las posibilidades jurídicas está determinado a través de principios y reglas que juegan en sentido contrario.

En cambio, las reglas son normas que exigen un cumplimiento pleno y, en esa medida, pueden siempre ser sólo o cumplidas 
o incumplidas. Si una regla es válida, entonces es obligatorio hacer precisamente lo que ordena, ni más ni menos. Las reglas contienen por ello determinaciones en el campo de lo posible fáctica y jurídicamente. Lo importante por ello no es si la manera de actuar a que se refiere la regla puede o no ser realizada en distintos grados. Hay por tanto distintos grados de cumplimiento. Si se exige la mayor medida posible de cumplimiento en relación con las posibilidades jurídicas y fácticas, se trata de un principio. Si sólo se exige una determinada medida de cumplimiento, se trata de una regla.

\section{1. 5. Los principios y la separación entre Derecho y moral}

No es difícil reconocer que la presencia de principios, por tanto, de mandatos de optimización, en el sistema jurídico tiene consecuencias en cuanto al carácter de éste y al concepto de Derecho, que sobrepasan con mucho el aspecto metodológico. Donde esto es más claro es en los principios constitucionales, como los de dignidad humana, libertad, igualdad, democracia, Estado de Derecho y Estado social. Si una constitución contiene estos seis principios, ello significa que se han incorporado a ella las formas principales del Derecho racional de la modernidad. El carácter de los principios significa que no se trata simplemente de normas vagas, sino que con ellas se plantea una tarea de optimización. Dicha tarea es, en cuanto a la forma, jurídica; en cuanto al fondo, sin embargo, es siempre también moral, a causa de su contenido moral. Puesto que algo análogo vale para muchos otros principios, la teoría de los principios ofrece un punto de partida adecuado para atacar la tesis positivista de la separación entre Derecho y moral.

El problema de la relación entre Derecho y moral ha de plantearse de nuevo bajo el aspecto de la vinculación entre la argumentación jurídica y la moral. Sobre la base del concepto de principio obtenido cabe preguntar ante todo si es posible una teoría de los principios que determine para cada caso justamente una respuesta.

\section{2. Teoría de los principios y única respuesta correcta}

\section{2. 1. Teorías fuertes y débiles de los principios}

Supongamos que se pudiera crear una lista en cierto modo completa de los principios de un sistema jurídico. Puesto que no 
contendría nada sobre el peso relativo de los principios, es decir, sobre la relación de prioridad entre ellos, ciertamente podría decirnos qué es lo que hay que tomar en consideración, pero no qué es lo que tiene preferencia en cuanto al resultado. Por ello, no sería mucho más que un catálogo de puntos de vista o de topoi. Esta simple relación de principios representa, desde el punto de vista de su rendimiento, la variante más débil de una teoría de los principios. La variante más fuerte sería una teoría que contuviera, además de todos los principios, todas las relaciones de prioridad abstractas y concretas entre ellos y, por ello, determinara unívocamente la decisión en cada uno de los casos. Si fuera posible una teoría de los principios de la forma más fuerte, sería sin duda acertada la tesis de Dworkin de la única respuesta correcta.

\section{2. 2. Teoría de los principios y teoría de los valores}

Para descubrir lo fuerte que pueda ser una teoría de los principios desde el punto de vista de su rendimiento, hay que fijarse en la semejanza que tienen los principios con lo que se denomina «valor». En lugar de decir que el principio de la libertad de prensa colisiona con el de la seguridad exterior, podría decirse que existe una colisión entre el valor de la libertad de prensa y el de la seguridad exterior. Toda colisión entre principios puede expresarse como una colisión entre valores y viceversa. La única diferencia consiste en que en la colisión entre principios se trata de la cuestión de qué es debido de manera definitiva, mientras que la solución a una colisión entre valores contesta a qué es de manera definitiva mejor. Principios y valores son por tanto lo mismo, contemplado en un caso bajo un aspecto deontológico, y en otro caso bajo un aspecto axiológico. Esto muestra con claridad que el problema de las relaciones de prioridad entre principios se corresponde con el problema de una jerarquía de los valores.

\section{2. 3. Orden estricto}

Los problemas de una jerarquía de los valores jurídicamente relevantes se han discutido con frecuencia. Se ha mostrado así que no es posible un orden que conduzca en cada caso precisamente a un resultado -a tal orden habría que llamarlo «orden estricto». Un orden estricto solamente sería posible si el peso de 
los valores o de los principios y sus intensidades de realización fueran expresables en una escala numérica, de manera calculable. El programa de semejante orden cardinal fracasa ante los problemas de una medición del peso y de la intensidad de realización de los principios jurídicos o de los valores jurídicos, que sea más que una ilustración de un resultado ya encontrado.

\section{2. 4. Orden débil}

El fracaso de los órdenes estrictos no significa sin embargo que sean imposibles teorías de los principios que sean más que un catálogo de topoi. Lo que es posible en un orden débil que consista de tres elementos: 1) un sistema de condiciones de prioridad, 2) un sistema de estructuras de ponderación y 3 ) un sistema de prioridades prima facie.

\section{2. 4. 1. Condiciones de prioridad}

El que las colisiones entre principios deban resolverse mediante ponderación en el caso concreto, no significa que la solución de la colisión sea solamente significativa para el caso concreto. Antes bien, pueden establecerse, con ocasión de la decisión para casos concretos, relaciones de prioridad que son importantes para la decisión de nuevos casos.

Un ejemplo lo ofrece el caso Lebach del Tribunal Constitucional alemán, en el que se trataba de una información televisiva repetida, no amparada ya por un interés informativo actual, sobre un delito grave, emitida poco antes de la excarcelación del autor ${ }^{3}$. La argumentación del tribunal se desarrolla en tres niveles. En el primer nivel constata una colisión entre la protección fundamental e igual de la personalidad y la libertad de información. En el segundo nivel establece que bajo una condición determinada y relativamente abstracta, esto es, la de una información actual sobre un delito grave, existe una prioridad de principio o prima facie en favor de la libertad de información. En el tercer nivel decide finalmente que bajo cuatro condiciones que se dan en el caso Lebach, a saber, una 1) información televisiva repetida, 2) no amparada ya por un interés informativo, 3) sobre un delito grave, 4) que pone en peligro la resocialización del autor, la protección de la personalidad prevalece sobre la

\footnotetext{
${ }^{3}$ BverfGE, 35, 202.
} 
libertad de información. Esto significa que tienen validez las consecuencias jurídicas del principio prevaleciente y que, por tanto, la emisión del programa televisivo está prohibido bajo estas cuatro condiciones. Lo último lleva a una idea fundamental para la relación de los niveles de la regla y de los principios, que se puede formular en una ley de colisión: las condiciones, bajo las que un principio prevalece sobre otro, forman el supuesto de hecho de una regla que determina las consecuencias jurídicas del principio prevaleciente.

Las condiciones de prioridad establecidas hasta el momento en un sistema jurídico y las reglas que se corresponden con ellas proporcionan información sobre el peso relativo de los principios. Sin embargo, a causa de la posibilidad de nuevos casos con nuevas combinaciones de características, no se puede construir con su ayuda una teoría que determine para cada caso precisamente una decisión. Pero de todos modos, abren la posibilidad de un procedimiento de argumentación que no se daría sin ellas. Este procedimiento, desde luego, debe ser incluido en una teoría completa de la argumentación jurídica.

\section{2. 4. 2. Estructuras de ponderación}

En contra del concepto de una teoría de los principios basada en condiciones de prioridad podría hacerse valer que, puesto que las condiciones de prioridad implican reglas, sólo se necesitaría tomar en consideración estas reglas. Esta objeción lleva a un segundo elemento fundamental de la teoría débil de los principios, a la ley de la ponderación. Los principios, en cuanto mandatos de optimización, exigen una realización lo más completa posible, en relación con las posibilidades jurídicas y fácticas. La referencia a las posibilidades fácticas lleva a los bien

conocidos principios de adecuación y necesidad. La referencia a las posibilidades jurídicas implica una ley de ponderación que puede ser formulada como sigue: Cuanto más alto sea el grado de incumplimiento o de menoscabo de un principio, tanto mayor debe ser la importancia del cumplimiento del otro. La ley de ponderación no formula otra cosa que el principio de la proporcionalidad en sentido estricto. Con ello se dice, grosso modo (lo que se puede demostrar de manera exacta) que el principio de proporcionalidad, con sus tres principios parciales ya mencionados, se sigue lógicamente del carácter principal de las normas, y éste de aquél. Esto no es sólo interesante para un examen teórico-estructural. Significa que una teoría de los principios conduce 
a estructuras de argumentación racional, lo que no vale para un simple catálogo de topoi. A pesar de esta contribución a la racionalidad práctica que no hay que infravalorar, es, sin embargo, también clara la urgencia con que la teoría de los principios necesita un complemento a través de una teoría de la argumentación jurídica de mayor alcance.

\section{2. 4. 3. Prioridades prima facie}

El tercer elemento de una teoría débil de los principios son las prioridades prima facie. Ya se mencionó un ejemplo. En una información actual sobre un delito grave, a la libertad de información le corresponde una prioridad prima facie frente a la protección de la personalidad. Las prioridades prima facie establecen cargas de la argumentación. De esta manera crean un cierto orden en el campo de los principios. Desde luego, no contienen una determinación definitiva. Si son más fuertes los argumentos en favor de una prioridad de un principio que juega en sentido contrario, se cumple suficientemente con la carga de la prueba. Con ello, el orden depende de nuevo de la argumentación.

\section{Argumentación jurídica y razón práctica}

\section{1. Un modelo de tres niveles del sistema jurídico}

Las reflexiones anteriores muestran que es imposible una teoría fuerte de los principios de forma que determine para cada caso precisamente una respuesta. Esto es así no sólo a causa de la limitación del poder del conocimiento humano, que podría superar un Hércules dworkiano, sino también por razones lógicas en sentido más amplio. Pero de todos modos es posible una teoría débil de los principios. Esta, sin embargo, no determina para cada caso una única respuesta correcta. Sin embargo, la idea de una única respuesta correcta no tiene por que ser abandonada. Se puede seguir manteniendo por dos vías. La primera la recorre quien afirma que la existencia de una única respuesta correcta no depende de que exista un procedimiento que permita mostrarlo. Esta no se seguirá aquí. La segunda vía se abre con la comprensión de que la adición de un nivel de los principios al de las reglas no lleva todavía a un modelo completo del sistema jurídico. Los principios, como las reglas, no regulan por sí mismos su aplicación. Si se quiere lograr un modelo adecuado del 
sistema jurídico, entonces se debe añadir a estos dos niveles que expresan, en relación con la cuestión de la corrección de la decisión, el lado pasivo del sistema jurídico, otro lado activo referido a esta cuestión. Los niveles de la regla y de los principios deben ciertamente complementarse con un tercero, a saber, con una teoría de la argumentación jurídica, que dice cómo, sobre la base de ambos niveles, es posible una decisión racionalmente fundamentada. Una respuesta concluyente a la cuestión de la única respuesta correcta sólo es posible refiriéndose a estos tres niveles.

\section{2. Supuestos básicos de la teoría de la argumentación jurídica}

El punto de partida de la teoría de la argumentación jurídica es que en la jurisprudencia se trata en definitiva siempre de cuestiones prácticas, por tanto, de lo que está ordenado, prohibido y permitido. La argumentación jurídica es por ello un caso especial de la argumentación práctica en general. Es un caso especial porque está situada bajo una serie de vínculos institucionales que brevemente pueden caracterizarse como la vinculación a la ley, al precedente y a la dogmática. Estos vínculos, que pueden concebirse mediante un sistema de reglas y formas específicas de la argumentación jurídica, no llevan sin embargo en cada caso precisamente a un resultado. Esto vale tanto para la subsunción bajo reglas como para la ponderación de principios. Por lo que respecta a los principios, ha quedado claro que su estructura, en cuanto mandatos de optimización, lleva a que, siempre que exhiban un contenido moral, en su aplicación haya que contestar siempre cuestiones morales. Este es un aspecto de la tesis general de que en todos los casos más o menos problemáticos son necesarias valoraciones que no pueden extraerse obligatoriamente del material autoritativamente fijado. La racionalidad de la argumentación jurídica depende por ello esencialmente de sí, y con qué alcance, estas valoraciones adicionales son susceptibles de un control racional.

\section{3. Teoría procedimental}

La cuestión de la racionalidad de la fundamentación jurídica lleva así a la cuestión de la posibilidad de fundamentar racionalmente los juicios prácticos o morales en general. La discusión de esta cuestión se ha visto obstaculizada largo tiempo por la oposición entre dos posiciones básicas extremas de las que siempre 
se presentan nuevas variantes: por un lado, las posiciones subjetivas, relativistas, decisionistas $\mathrm{y} / \mathrm{o}$ irracionalistas $\mathrm{y}$, por otro lado, las posiciones objetivistas, absolutistas, cognocistivistas y/o racionalistas. No existe sin embargo ningún motivo para una semejante actitud todo-o-nada. Ciertamente no son posibles teorías morales materiales que para cada cuestión práctica permitan extraer con seguridad intersubjetivamente concluyente precisamente una respuesta, pero sí que son posibles teorías morales procedimentales que formulan reglas o condiciones de la argumentación o decisión práctica racional. Una versión especialmente prometedora de una teoría moral procedimental es la del discurso práctico racional.

\section{4. Discurso, única respuesta correcta, razón práctica}

\section{4. 1. Reglas del discurso}

La pieza nuclear de la teoría del discurso está formada por un sistema de reglas del discurso y de principios del discurso, cuya observancia asegura la racionalidad de la argumentación y de sus resultados. En otro lugar he tratado de formular explícitamente este sistema a través de un sistema de 28 reglas. Las reglas se extienden desde aquéllas que exigen no contradicción, claridad lingüística y verdad empírica, pasando por aquéllas que expresan la idea de universalizabilidad, entre otras cosas asegurando a cada uno el derecho a tomar parte en el discurso y a cada uno la misma consideración en el discurso, hasta aquéllas que rigen para la argumentación consecuencialista, la ponderación y el análisis de la formación de convicciones normativas. Este sistema de reglas que pretende formular algo así como un código de la razón práctica, no sólo complementa las reglas específicas del discurso jurídico, sino que constituye también la base para su justificación y crítica, en el marco de una justificación y crítica del sistema jurídico en su conjunto.

\section{4. 2. Única respuesta correcta}

La cuestión de la única respuesta correcta depende esencialmente de si el discurso práctico lleva a una única respuesta correcta para cada caso. Llevaría a ello si su aplicación garantizara siempre un consenso. Ya un simple esbozo muestra claramente que varias de sus exigencias, bajo condiciones reales, sólo se 
pueden cumplir de manera aproximada. Esto ya excluye un consenso para cada cuestión. Por tanto, una única respuesta correcta para cada caso sólo podría admitirse recurriendo a cinco idealizaciones, a saber: 1) tiempo ilimitado, 2) información ilimitada, 3) claridad lingüística conceptual ilimitada, 4) capacidad y disposición ilimitada para el cambio de roles y 5) carencia de prejuicios ilimitada.

\section{4. 3. Razón y corrección relativa}

Aquí queda aún por ver si sobre la base de semejantes idealizaciones es posible una única respuesta correcta para cada caso o si las diferencias antropológicas de los participantes en el discurso que obstaculizan el discurso hacen que, pese a las idealizaciones, puedan existir casos sin una única respuesta correcta. En todo caso, está claro que en la realidad no existe ningún procedimiento que permita, con una seguridad intersubjetivamente necesaria, llegar en cada caso a una única respuesta correcta. Esto último no obliga sin embargo a renunciar a la idea de la única respuesta correcta, sino que únicamente da ocasión para determinar su status con más precisión. El punto decisivo aquí es que los respectivos participantes en un discurso jurídico, si sus afirmaciones y fundamentaciones han de tener un pleno sentido, deben, independientemente de si existe o no una única respuesta correcta, elevar la pretensión de que su respuesta es la única correcta. Esto significa que deben presuponer la única respuesta correcta como idea regulativa. La idea regulativa de la única respuesta correcta no presupone que exista para cada caso una única respuesta correcta. Sólo presupone que en algunos casos se puede dar una única respuesta correcta y que no se sabe en qué casos es así, de manera que vale la pena procurar encontrar en cada caso la única respuesta correcta. Las respuestas que se encuentren, en el marco de este intento, sobre la base del nivel de la regla y de los principios, de acuerdo con los criterios de la argumentación jurídica racional, que incluyen los de la argumentación práctica general, también responden entonces, aunque no sean las únicas respuestas correctas, a las exigencias de la razón práctica y, en este sentido, son al menos relativamente correctas.

(Trad. de Manuel Atienza) 